\title{
Auditoría de Gestión Administrativa aplicada a la Unidad Educativa Fiscal "Alejo Lascano" en la Ciudad de Jipijapa
}

\section{Audit of Administrative Management applied to the Fiscal Education Unit "Alejo Lascano" in the City of Jipijapa}

\section{Auditoria de Gestão Administrativa aplicada à Unidade de Educação Fiscal "Alejo Lascano" na cidade de Jipijapa}

Miguel E. Pionce-Soledispa ${ }^{\mathrm{I}}$

miedpiso@hotmail.com

Recibido: 27 de junio de 2018 * Corregido: 28 de agosto de 2018 * Aceptado: 15 de septiembre de 2018

I. Magister en Auditoria Gubernamental y Control, Magister en Gerencia Educativa, Ingeniero en Auditoria, Licenciado en Análisis de Sistemas. Especialista nacional de control patrimonial en Contraloría General del Estado, Universidad Estatal del Sur de Manabí Jipijapa; Ecuador. 


\section{Resumen}

El objetivo principal de este estudio fue realizar una auditoría de gestión en la Unidad Educativa Fiscal "Alejo Lascano" de la ciudad de Jipijapa, con el propósito de determinar el grado de cumplimiento de las metas, objetivos y el nivel de eficiencia, eficacia, economía, en la utilización de sus recursos, en base al cumplimiento de políticas o normativa que rige a la institución, esto a través de indicadores de gestión. Metodológicamente se apoyó en métodos de investigación como el deductivo, inductivo y analítico así como técnicas de encuesta y entrevista que ayudaron a determinar los problemas en la institución educativa. La auditoría de gestión administrativa fue aplicada siguiendo la guía metodológica para la Auditoria de Gestión expedida mediante Acuerdo Ministerial 047-CG-22011 por parte de la Contraloría General del Estado, para lo cual se realizó una planificación preliminar, un programa de auditoría, para luego aplicar el programa y finalmente emitimos el informe respectivo que se presentó al Rector.

Palabras claves: Evaluación, indicadores de gestión, planificación preliminar, eficiencia, eficacia, economía. 


\section{Abstract}

The main objective of this study was to conduct a management audit in the Educational Unit "Alejo Lascano" of the city of Jipijapa, with the purpose of determining the degree of compliance with the goals, objectives and the level of efficiency, effectiveness, economy, in the use of its resources, based on compliance with policies or regulations that govern the institution, this through management indicators. Methodologically, it relied on research methods such as deductive, inductive and analytical methods as well as survey and interview techniques that helped determine the problems in the educational institution. The audit of administrative management was applied following the methodological guide for the Management Audit issued by Ministerial Agreement 047-CG-22011 by the Comptroller General of the State, for which a preliminary planning was carried out, an audit program, for later apply the program and finally we issued the respective report that was presented to the Rector.

Key words: Assessment, management indicators, preliminary planning, efficiency, efficiency, economy. 


\section{Introducción.}

La administración es la orientación eficaz de actividades que se realizan en una Institución con la participación de otras personas, con el firme propósito de obtener resultados eficientes y eficaces. La Auditoría de Gestión en el ámbito administrativo de las instituciones públicas juega un rol muy importante debido a que permite pronunciarse sobre la legalidad, transparencia y eficiencia relacionada a las actividades que el personal que labora en las instituciones, realiza en los distintos departamentos.

En este orden de ideas, la presente investigación estuvo orientada al estudio de las actividades administrativas y al cumplimiento de políticas o normativas que rigen a la Institución, esto a través de la utilización de las Técnicas de Auditoría que ayudaron a determinar la eficiencia, eficacia, economía, con que se desarrollan las funciones asignadas.

La Auditoría como tal, existe desde tiempos remotos aunque no como se la conoce actualmente, debido a la inexistencia de sistemas contables complejos, pero esta es fundamental en las empresas privadas o públicas, aunque hoy en día su objeto de estudio se ha ampliado por los cambios sociales y tecnológicos y su implementación permitirá evaluar la gestión de los procesos que se realizan en una institución.

El control de la gestión en la administración de las empresas es necesario, por lo que se asegura la obtención de recursos y su empleo eficaz y eficiente en el cumplimiento de los objetivos, esto busca fundamentalmente la supervivencia y crecimientos de las entidades. 
En el libro titulado Principios de Administración (1 ${ }^{\mathrm{a}}$. Edición: julio de 2008) escrito por Darío Hurtado Cuartas se establece a la administración como:

Proceso que comprende funciones y actividades que los administradores deben llevar a cabo para lograr los objetivos de la organización. En este sentido, podríamos decir que la administración es la conducción racional de actividades, esfuerzos y recursos de una organización, resultándole algo imprescindible para su supervivencia y crecimiento, asi mismo Henry Fayol la administración puede verse como un proceso, dicho proceso está compuesto por funciones básicas: planificación, organización, dirección, coordinación, control. (Hurtado Cuartas , 2008)

Del mismo modo, B Jaramillo en su libro nombrado Auditoría Administrativa: Gestión Estratégica del Cambio (2007). Comento lo siguiente "Una auditoría administrativa es la revisión analítica total o parcial de una organización con el propósito de precisar su nivel de desempeño y perfilar oportunidades de mejora para innovar valor y lograr una ventaja competitiva sustentable" (p. 11) (Beltrán Jaramillo, 2010).

Así mismo como explica, Mora A. en su libro titulado. Diccionario de Contabilidad, Auditoría y Control de Gestión (2008) el manifiesta que la auditoría de gestión es una “Actividad de auditoría que tiene por objeto la verificación del proceso de toma de decisiones de una entidad, con el propósito de alcanzar los propósitos y objetivos marcados para la misma.” (Mora Enguídanos, 2008)

Por su parte Según la Contraloría General del Estado en su Manual de Auditoria de Gestión; 2003, define a la gestión como: "proceso mediante el cual la entidad asegura la obtención de 
recursos y su empleo eficaz y eficiente en el cumplimiento de sus objetivos". (Contraloría General del Estado, 2003)

En este mismo sentido: "La asunción y ejercicio de responsabilidades sobre un proceso (es decir, sobre un conjunto de actividades) lo que incluye: La preocupación por la disposición de los recursos y estructuras necesarias para que tenga lugar, la coordinación de sus actividades (y correspondientes interacciones) y la rendición de cuentas ante el abanico de agentes interesados por los efectos que se espera que el proceso desencadene" (Fontana, 2009)

\section{La Auditoría de Gestión}

La auditoría de gestión avalúa la eficiencia, eficacia, economía, ética y ecología en cuanto a la planeación estratégica propia de la organización, revisa y analiza si los procesos realizados en toda la institución van de la mano con los objetivos estratégicos planteados (Blanco Luna, 2012).

La auditoría de gestión para medir la utilización óptima de sus recursos utiliza indicadores, los cuales reflejaran el tiempo estimado para cumplir con sus objetivos y metas establecidos.

Además, la auditoría asesora a la gerencia sobre qué medidas tomar en caso de encontrarse falencias, es decisión de la gerencia aceptar y aplicar estar recomendaciones, caso contrario, puede escoger otro tipo de sugerencias que la crea conveniente, siempre que sirva para solucionar dichas fallas (Blanco Luna, 2012).

(Estupiñán Gaitán, 2011), manifiesta que la auditoría de gestión examina en forma detallada cada aspecto operativo de la organización, por lo que, en la determinación del alcance debe considerarse lo siguiente: 
- "Logro de los objetivos institucionales; nivel jerárquico de la entidad; la estructura organizativa; y, la participación individual de los integrantes de la institución.

- Verificación del cumplimiento de la normatividad tanto general como específica y de procedimientos establecidos.

- Evaluación de la efíciencia y economía en el uso de los recursos, entendido como rendimiento efectivo, o sea operación al costo mínimo posible sin desperdicio innecesario; así como, de la eficacia en el logro de los objetivos y metas, en relación a los recursos utilizados.

- Medición del grado de confiabilidad, calidad y credibilidad de la información financiera y operativa.

- Atención a la existencia de procedimientos ineficaces o más costosos; duplicación de esfuerzos de empleados u organizaciones; oportunidades de mejorar la productividad con mayor tecnificación; exceso de personal con relación al trabajo a efectuar; deficiencias importantes, en especial que ocasionen desperdicio de recursos o perjuicios económicos.

- Sobre el alcance de la auditoría, debe existir acuerdo entre los administradores y auditados; el mismo debe quedar bien definido en la fase de Conocimiento Preliminar, porque permite delimitar el tamaño de las pruebas o sea la selección de la muestra y el método aplicable, además medir el riego que tiene el auditor en su trabajo" (Estupiñán Gaitán, 2011) 
Según (Vásquez Miranda, 2010), en su libro Control fiscal y auditoría de estado en Colombia, señala que el alcance de la auditoría es: "Una derivación de su objetivo es decir determina el tipo de labor que debe realizarse”. Pág. 90

(Blanco Luna, 2012), en su libro titulado. Auditoría integral, normas y procedimientos p .45. Mencionó que la auditoría de gestión tendría el siguiente alcance:

- "Verificar la existencia de objetivos y planes coherentes y realistas.

- Comprobar la confiabilidad de la información que se toma de base para el cálculo de los indicadores de gestión.

- Verificar la existencia de métodos o procedimientos adecuados de operación y la eficiencia de los mismos.

- Comprobar la utilización adecuada de los recursos importantes de la compañía.

- Evaluar la estructura organizacional de la compañía.” (Blanco Luna, 2012)

De acuerdo a los criterios anteriores, el alcance de la auditoría de gestión se refiere a los procesos determinados para lograr el objetivo del examen que se realiza a una organización, como el análisis de la misión y visión institucional, del uso adecuado de los recursos, su buena organización institucional, entre otros.

\section{Materiales y métodos.}

La investigación ha sido realizada tomando en cuenta las fuentes de información, procediendo metodológicamente, analizando los datos e información obtenida de la Comunidad 
Educativa “Alejo Lascano", sujeto de la presente investigación. Considerando la parte bibliográfica como un elemento de suma importancia en nuestra investigación, se aplicó el método bibliográfico y con él se obtuvo toda la bibliografía referente a éste tema de investigación, la misma que se pudo confrontar con los datos de la investigación de campo.

Se aplicó la modalidad de campo con el fin de obtener información de acuerdo con los objetivos del proyecto, de esta manera se pudo tomar contacto en forma directa con la realidad, se aplicó entrevista a los Directivos de la Unidad Educativa Fiscal "Alejo Lascano" y encuestas a los Profesores y personal administrativo de la Institución Educativa. A través del método estadístico se realizó la tabulación de las encuestas aplicada, la representación gráfica de las mismas como también permitió determinar la muestra.

Para el análisis de la información recabada se emplearon técnicas de investigación primaria como la entrevista aplicada a los Directivos de la Unidad Educativa Fiscal "Alejo Lascano" y la encuesta aplicada a 56 docentes y 5 personal administrativo.

\section{Resultados.}

\section{De la auditoria de gestión administrativa.}

\section{Plan estratégico.}

De la visita efectuada a la Institución, así como la verificación mediante la evaluación de Control Interno, se establece que la Unidad Educativa Fiscal "Alejo Lascano" no cuenta con un plan estratégico. 
La norma de control interno 200-04: Estructura organizativa que establece que la máxima autoridad debe crear una estructura organizativa que atienda el cumplimiento de su misión y apoye efectivamente el logro de los objetivos organizacionales, la realización de los procesos, las labores y la aplicación de los controles pertinentes.

\section{Conclusión.}

La Unidad Educativa Fiscal “Alejo Lascano”, no se ha observado la técnica moderna de un plan estratégico que permite dar una orientación hacia donde se dirige la Institución por lo que toda la gestión administrativa y financiera se está realizando empíricamente.

\section{Recomendación.}

\section{Al Rector de la Institución.}

Deberá reunirse con los miembros del Consejo Ejecutivo y discutir nuevos cambios en el plan estratégico que posee en la actualidad.

\section{Manual de procedimientos contables.}

Del análisis de control interno, se determinó que la Institución no cuenta con un manual de procedimientos administrativos; mismos que mejorarán la organización y ordenamiento de la gestión, llevada por la Unidad Educativa.

Sin embargo, la administración de la Unidad Educativa no considera necesario implementar un manual de procedimientos, ya que utilizan el reglamento de la LOEI. Este hecho puede ocasionar cruces de información y pérdidas de tiempo al momento de requerir algún tipo de información. 


\section{Conclusión.}

La falta de un manual de procedimientos afecta a las actividades realizadas por la Institución, cuando existan problemas de cruce de información

\section{Recomendación.}

\section{Al Rector de la Institución.}

Es necesario implementar un manual de procedimientos administrativos y dar a conocer a todos el personal administrativo y operativo, de esta manera se llevara un mejor orden en el manejo de información y responsabilidades.

\section{Control de asistencias.}

De la visita a la Institución y la evaluación de control interno, se determinó que la Unidad Educativa supervisa los horarios de entrada y salida de los empleados y trabajadores mediante hojas de control.

La norma de control interno 407-09: Asistencia y permanencia del personal que establece la administración de personal de la entidad establecerá procedimientos y mecanismos apropiados que permitan controlar la asistencia y permanencia de sus servidoras y servidores en el lugar de trabajo

\section{Conclusión.}

La inexistencia de un sistema de control digital de asistencias, puede causar irresponsabilidad por parte de los trabajadores, ya que tal vez no estén cumpliendo con su horario respectivo. 


\section{Recomendación}

\section{Al Jefe de la Unidad de Administración de Talento Humano.}

Buscar los mecanismos antes el Distrito de Educación para implementar un sistema de control digital de asistencia con el fin de mantener el orden y la disciplina de sus trabajadores

\section{Etiquetas de códigos de barras en los activos fijos.}

De las visitas realizadas a la institución se observó que los activos fijos no están debidamente codificados. El marco legal educativo establece que se debe llevar un inventario actualizado del mobiliario y demás activos fijos y para agilitar el proceso de actualización de inventarios es recomendable utilizar un sistema de codificación. El Rector menciono que la administración y control de inventarios de activos fijos es explícitamente una función del administrador del circuito educativo.

\section{Conclusión.}

Inexistencia de codificación de activos fijos debido al descuido por parte de la administración en fijar un mayor control en los bienes de la institución.

\section{Recomendación.}

\section{Al Rector de la Institución.}

Hacer los tramites respectivo antes el Distrito de Educación para que se invierta en tecnología de un sistema de codificación de activos que agilite los procesos del conteo físico ahorrando tiempo y dinero. 


\section{Indicadores de evaluación del comportamiento.}

La evaluación realizada al control interno de la Unidad Educativa Fiscal "Alejo Lascano" revelo que la Institución no cuenta con indicadores de rendimiento,

Incumplimiento del Art. 22 del Marco Legal Educativo, que establece la evaluación del comportamiento, lo cual cumple un objetivo formativo institucional a cargo de los integrantes de la Unidad Educativa.

La implementación de dichos indicadores contribuyen a la medición de las actividades ejecutadas en la entidad y también a eliminar deficiencias.

\section{Conclusión.}

La Institución Educativa no ve la importancia de aplicar indicadores de evaluación de la aplicación del código de convivencia.

\section{Recomendación.}

\section{Al Rector de la Institución.}

Se recomienda al Rector que debería implementar indicadores de rendimiento que mejoren los procesos ejecutados por la Unidad Educativa y contribuyan al logro de su desarrollo.

\section{Mecanismos para identificación de riesgos externos.}

Del análisis realizado al componente identificación de eventos se determinó que la Institución no cuenta con mecanismos que le permitan medir los sucesos externos que afectan a la misma. 
Las normas de control interno 300-01: Identificación de riesgos que determinan que los directivos de la entidad identificarán los riesgos que puedan afectar el logro de los objetivos institucionales debido a factores internos o externos, así como emprenderán las medidas pertinentes para afrontar exitosamente tales riesgos.

\section{Conclusión.}

La Unidad Educativa Fiscal "Alejo Lascano" no implementa mecanismos que identifiquen los posibles peligros, dependiendo del riesgo.

\section{Recomendación.}

\section{Al Vicerrector de la Institución.}

Analizar datos históricos en cuanto a los riesgos políticos del país y crear un plan de contingencia.

\section{Asignación adecuada del número de estudiantes.}

En la aplicación y análisis de indicadores se pudo identificar que la infraestructura de la institución no abastece el número de estudiantes que esta posee.

Según el artículo 156 numeral 1 del Marco Legal Educativo en caso de una sobredemanda de alumnos establece admitir a los estudiantes a través de un proceso de sorteo. Este problema se generó por la crisis de cupos en instituciones fiscales debido al cierre de varias de estas y también porque la administración no pudo identificar este tipo de riesgo que perjudicó el curso normal de la unidad educativa. 


\section{Conclusión.}

La Unidad Educativa no tiene la capacidad física para abastecer el cupo de estudiantes de la propia institución y de otras que fueron cerradas, lo que provoca exceso de demanda y por ende un ambiente laboral malo para estudiantes y docentes.

\section{Recomendación.}

\section{Al Rector de la Institución.}

Aplicar el reglamento del Marco Legal, para evitar sanciones, es decir la admisión a través de un proceso de sorteo; y a largo plazo solicitar la inversión para la construcción de más aulas.

\section{Cargos directivos con los perfiles requeridos por la ley.}

En el análisis de la documentación del perfil profesional de los directivos se encontró que Vicerrector no ha aprobado el programa de formación de directivos, requisito importante para acceder a estos cargos

Los artículos 120 y 121 del Marco Legal Educativo el cual menciona que para funcionar como Vicerrector debe haber aprobado el programa de formación de directivos. Sin embargo, esto puede provocar una mala gestión en sus actividades, ya que no está totalmente capacitado en su formación como directivo, pese a que ellos están al tanto de sus funciones asignadas en la Ley, no es suficiente para cumplir a cabalidad con un buen desempeño como autoridad educativa. 


\section{Conclusión.}

El Vicerrector de la institución cumple sus funciones solo como personal encargado ya que aún no han aprobado el programa de formación de directivos.

\section{Recomendación.}

\section{Al Rector de la Institución.}

Incentivar al personal profesional a llevar a cabo el proceso de promoción de docentes a Autoridad siendo estos el personal idóneo que acrediten las categorías escalafonarias establecidas en la ley.

\section{Registró de uso de recursos didácticos.}

Al ejecutar la auditoría de gestión administrativa se solicitó un detalle de los recursos didácticos que utilizan los docentes para impartir su clase pero no existía control alguno, pese a que una de las obligaciones de los miembros de la comunidad educativa es "Respetar y cuidar las instalaciones y recursos educativos...” así lo establece el artículo 18 literal c del Marco Legal Educativo.

\section{Conclusión.}

Desinterés en llevar un registro de uso de recursos didácticos, debido al exceso de confianza con los docentes por parte de las autoridades generando así una evasión de responsabilidad en caso de daño al bien. 


\section{Recomendación.}

\section{Al Rector de la Institución.}

Delegar a un funcionario de la Entidad a llevar un registro detallado de los bienes entregados a los docentes o estudiantes; con nombre, fecha de entrega, hora, nombre o código del recurso y firma del responsable.

\section{Código de ética.}

Se pudo determinar que no cuenta con un código de ética; el mismo que es una guía conductora para las personas que laboran en la Unidad Educativa

La norma de control interno 200-01 establece que la máxima autoridad y los directivos establecerán los principios y valores éticos como parte de la cultura organizacional para que perduren frente a los cambios de las personas de libre remoción; estos valores rigen la conducta de su personal, orientando su integridad y compromiso hacia la organización. Esto se originó ya que la institución cuenta con un código de convivencia al cual se lo confundió con el código de ética.

\section{Conclusión.}

La institución no ve la importancia de aplicar indicadores de evaluación de la aplicación del código de convivencia

\section{Recomendación.}

\section{Al Consejo Ejecutivo.}


Se recomienda al Consejo Ejecutivo de la Institución Educativa realizar un código de ética interno, que incluya valores, principios y responsabilidades.

\section{Discusión.}

En base a los resultados obtenidos se hace referencia a lo indicado por Cuenca Merino Irlanda Maritza y Lapo Guamán Diana Maribel (2011), establecieron en su tesis titulada Auditoria de Gestión en la Unidad Educativa Fiscomisional "María Auxiliadora" de la ciudad de Cariamanga concluyendo en lo siguiente:

Se determinó la eficiencia, eficacia y calidad en los que se resaltan las diferentes funciones que se realizan en la Institución Educativa, para la aplicación de los programas de auditoría se aplicaron métodos y técnicas que ayudó a obtener las evidencias y hallazgos, proporcionando así una alternativa de solución a la Institución. La calidad de educación según el criterio de padres de familia y estudiantes encuestados la calificación de muy buena, debido al cumplimiento de las planificaciones, responsabilidad y metodología utilizada en 1 institución. (Cuenca Merino \& Lapo Guamán, 2011)

Del mismo modo Karla Alexandra Saavedra Merino (2011), estableció en su tesis titulada Auditoria de Gestión al área administrativa de la Unión de Cooperativa de Transporte en Taxis Azuay:

Las funciones del personal administrativo no están claramente definidas en el estatuto, es por ello que el Gerente no puede delegar las diferentes actividades al personal encargado ya que esto se debe a que no existe un parámetro en la cual indique la actividad respectiva para cada empleado, por esta razón se debe actualizar inmediatamente los reglamentos de la entidad para que el Gerente no 
esté desempeñando funciones que no están a su cargo. En la actualidad la entidad no cuenta con un control adecuado con relación al control de asistencia, esto se debe a la falta de actualización de las leyes ya que no está establecido un parámetro en el que indique cuáles son las actividades que cada miembro debe cumplir, es por este motivo que trae como efecto un riesgo financiero por los costos generados en los tiempos improductivos. (Saavedra Merino, 2011)

Igualmente Fanny Almeida Velasco (2008), estableció en su tesis titulada Auditoria de Gestión Administrativa del área Capacitación del Instituto de Capacitación Municipal:

La Institución no cuenta con el "Manual de Capacitación y Desarrollo de Talento Humano del Municipio" establecido en el instructivo de aplicación de la Carrera Administrativa Municipal, consecuentemente no se estableció políticas, normas y procedimientos de capacitación ni se legalizó el Reglamento de Capacitación situación que no permitió que el personal sujeto a la LOSEP cuente con los requisitos de capacitación. Las acciones necesarias para para el cumplimiento y lograr lo planificado dentro del Marco de la Misión, Visión y Objetivos Estratégicos no se ejecutaron, lo que originó que la acción institucional se ejecute de manera limitada y desviada de la realidad. (Almeida Velasco, 2006)

\section{Conclusiones.}

Mediante la aplicación de la auditoría de gestión administrativa a la Unidad Educativa Fiscal "Alejo Lascano" se pudo evaluar, conocer e identificar las falencias en el control interno y proporcionar pautas necesarias para plantear posibles soluciones.

Una vez concluido el diagnóstico técnico situacional se llegó a determinar que el personal que labora en las institución conoce sus funciones y todas las actividades que debe desarrollar para 
cumplir el objetivo institucional, sin embargo dichas funciones, actividades y cargos no están establecidos en organigramas y manuales administrativos que guíen al desarrollo eficiente y correcto del desenvolvimiento de la Unidad Educativa.

Con la conceptualización de caracteres importantes en la auditoría se concluye que los responsables de la dirección de la institución desconocen aspectos fundamentales que guían al desarrollo de una buena administración y un adecuado control interno por lo que no se ejecutan distintos procesos en función a la normativa establecida.

Una vez concluida la ejecución del plan de auditoría se pudo establecer distintas recomendaciones en los informes generados las mismas que en su mayoría si se pusieron en práctica por su facilidad y tiempo de aplicación, generando de esta forma impactos positivos dentro de la Unidad Educativa.

\section{Recomendaciones.}

La auditoría de gestión administrativa representa una herramienta importante siempre y cuando se aplique las sugerencias dictadas por los auditores y no se vuelva a repetir las acciones que debilitan el desenvolvimiento y éxito de la institución.

La realización de un plan de capacitación, acerca de la normativa o conceptos desconocidos por el personal que labora en la unidad educativa, es uno de los puntos clave que atiende las necesidades de formación tanto del profesorado como de la institución.

La implementación de sugerencias establecidas en los informes emitidos de manera inmediata, contribuyen a la consecución de objetivos a mediano y largo plazo, haciendo uso 
adecuado de los recursos, mejorando el rendimiento de la administración, profesorado y por lo tanto el de los estudiantes.

Los informes emitidos en la auditoría de gestión es lo más relevante en esta investigación, los mismos que deben ser socializados a la junta directiva y autoridades de la institución, con el fin de que conjuntamente todos se comprometan a suprimir debilidad alguna y aprovechar fortalezas y oportunidades existentes en el medio que les rodea.

\section{Referencias Bibliográficas.}

Almeida Velasco, F. (2006). Auditoría de Gestión Administrativa del área Capacitación del Instituto de Capacitación Municipal. Quito: Universidad Tecnológica Equinoccial.

Beltrán Jaramillo, J. (2010). Indicadores de Gestión. Bogorá: Editores Ltda.

Blanco Luna, Y. (2012). Auditoría integral, normas y procedimientos. Bogota: Ecoe Ediciones.

Contraloría General del Estado. (2003). Manual de Auditoría de Gestión. Quito: CGE.

Cuenca Merino, I., \& Lapo Guamán, D. (2011). Aditoria de gestion en la Unidad Educativa Fiscomisional. Quito: UTPL.

Estupiñán Gaitán, R. (2011). Control Interno y fraudes con base a cilcos transaccionales. Bogotá: Ecoe Ediciones.

Fontana, F. (2009). Manual para la Gestión de la intervención social, políticas, organizaciones y sistemas para la acción. Madrid: CCS.

Hurtado Cuartas , D. (2008). Principios de Administración. Medellin : Instituto Tecnológico Metropolitano.

Mora Enguídanos, A. (2008). Diccionario de Contabilidad, Auditoría y Control de Gestión. Bogotá: ECOBOOK.

Saavedra Merino, K. (2011). Auditoría de gestión al área administrativa de la Unión de Cooperativas de Transporte en Taxis del Azuay (UCTTA). Quito: UPS.

Vásquez Miranda, W. (2010). Control Fiscal y Aditoría de Estado en Colombia. Bogotá: Universidad de Bogotá Jorge Tadeo Lozano. 